\title{
EL ANÁLISIS DE REDES COMPLEJAS Y LA
CONSERVACIÓN DE LA BIODIVERSIDAD
}

\section{Rico-Gray}

Departamento de Ecología Aplicada

Instituto de Ecología, A.C., Xalapa, Veracruz, México

En una comunidad cada especie interactúa de diferentes maneras con una o varias especies formando complejas redes de especies (JORDANO, 1987). La estructura de éstas es el resultado de un complejo de acciones recíprocas entre la biogeografía, los factores ecológicos (p.e., diferencias en abundancia entre especies) y los procesos coevolutivos (p.e., convergencia entre especies interactuantes) (BASCOMPTE et al., 2006). Una red de especies interactuantes puede tener un número reducido de enlaces entre las especies, indicando un ensamble de especialistas ecológicos, o muchos enlaces, indicando generalistas ecológicos (BASCOMPTE \& JORDANO, 2006). Uno de los problemas centrales a resolver en ecología de comunidades es si las diferentes formas de interacción o las diferentes condiciones ecológicas favorecen estructuras alternas en estas redes de especies interactuantes (GUIMARÃES et al., 2006). Los estudios comparativos sobre estructura de redes están ayudando a descubrir patrones de especialización ecológica a nivel de comunidad para diferentes tipos de interacciones interespecíficas (THOMPSON, 2006).

Las redes complejas no son usualmente homogéneas, así que podemos encontrar grupos de especies más conectados entre ellos que con el resto de las especies. Ciertas características generales de las redes se mantienen independientemente del tipo de comunidad estudiada (JORDANO et al., 2003). El entender tales propiedades es fundamental para entender la evolución de los ecosistemas y para el desarrollo de medidas viables de conservación (BASCOMPTE et al., 2003). Por ejemplo, la biodiversidad de una comunidad debe de incluir la lista con los nombres de las especies, pero también de sus interacciones. Se tiene un pequeño pero significativo grupo de investigaciones sobre cómo están estructuradas las comunidades de mutualistas ('mutualistic networks'; p.e., BASCOMPTE et al., 2003; JORDANO et al., 2003; BASCOMPTE \& JORDANO, 2006; BASCOMPTE et al., 2006; BLÜGTHEN et al., 2006; GUIMARÃES et al., 2006). Este enfoque puede llevar a plantear preguntas específicas sobre la organización de las comunidades, la variación geográfica y el proceso coevolutivo (THOMPSON, 2005).

Los mutualismos entre especies de vida libre muchas veces forman redes multiespecie donde las especies difieren en el número de especies con las que interactúan, creando redes complejas de interacciones que usualmente aparentan ser idiosincrásicas (BAS- 
COMPTE et al., 2003; JORDANO et al., 2003). Los estudios de interacciones entre polinizadores y plantas, frugívoros y frutos, y hormigas y plantas con nectarios sugieren cómo es que la especialización se distribuye entre las especies interactuantes (BASCOMPTE et al., 2003; JORDANO et al., 2003; VÁZQUEZ \& AIZEN, 2004; BASCOMPTE \& JORDANO, 2006; GUIMARÃES et al., 2006). BASCOMPTE et al. (2003) encontraron que estas redes usualmente exhiben un tipo específico de especialización asimétrica llamado 'nested' (encajado o anidado) (Figura 1). Las redes anidadas se caracterizan por (1) especies generalistas que interactúan entre
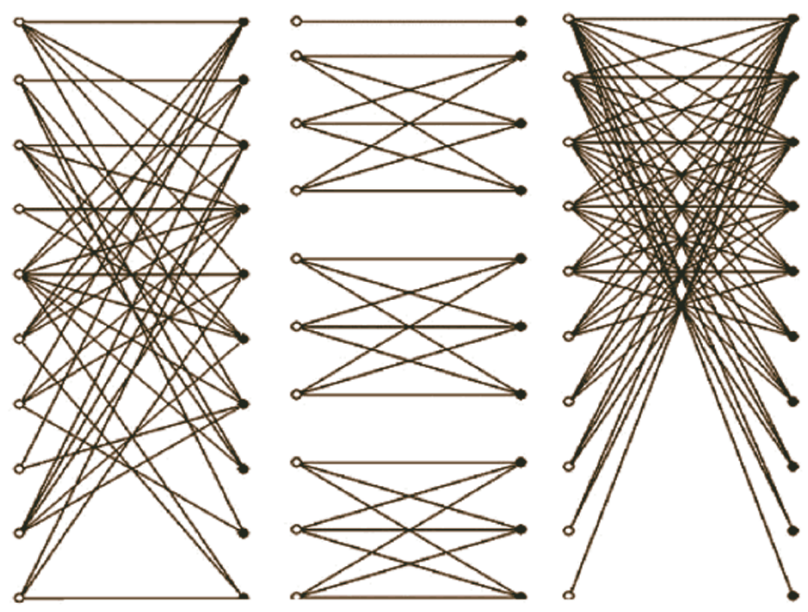

Figura 1. Tipos hipotéticos de redes planta-animal. De izquierda a derecha: al azar, compartimentada y anidada o encajada (GUIMARÁES et al., 2006).

ellas, formando un núcleo de especies interactuantes; (2) especies especialistas que comúnmente interactúan solamente con las generalistas, y (3) la ausencia de especialistas que interactúen solamente con otras especies especialistas (véase también VÁZQUEZ \& AIZEN, 2004) (Figura 2).

Se sabe muy poco sobre si las asimetrías en la especialización son comunes en otras formas de mutualismo, o cómo diferentes condiciones ecológicas pueden dar forma al grado de asimetría (o grado de anidación o de encajamiento) (Figura 3). Sabemos que las especies en los mutualismos comúnmente difieren geográficamente de las especies con las que interactúan (ANDERSON \& MAJER, 2004; RUD-
GERS \& STRAUSS, 2004; HERRERA, 2005) y que algunas interacciones coevolucionan como un mosaico geográfico en donde las poblaciones difieren a través de paisajes en su adaptación y su especialización con las otras especies (THOMPSON, 1994, 2005). El problema a resolver es si las redes de interacciones muestran patrones similares de especialización en diferentes comunidades sin importar qué especies particulares están involucradas. Al explorar la variación geográfica en patrones macroscópicos de redes entre mutualistas podremos asociar los dos principales enfoques para explorar la organización de mutualismos multiespecie: la teoría del mosaico geográfico y la teoría de las redes complejas (BASCOMPTE \& JORDANO, 2006).

Los resultados sugieren que la especialización es un carácter común de mutualismos no específicos y ricos en especies y que el tamaño de la red, la estructura de la vegetación y los aspectos idiosincrásicos de cada comunidad afectan el grado de anidación o encajamiento en mutualismos de vida libre (GUIMARÃES et al., 2006). Estos autores sugieren que la pérdida de biodiversidad en las comunidades biológicas puede tener efectos sobre la organización de estos mutualismos de baja especificidad, pero que la importancia de las especies generalistas puede variar geográficamente. Así, la distribución de especialistas y de generalistas en las redes no es al azar, la regla son las asimetrías en la especialización entre pares de especies. Una fuerte dependencia en una dirección es acompañada frecuentemente por una débil dependencia en la otra dirección (GUIMARÃES et al., 2006; BASCOMPTE et al., 2006; THOMPSON 2006).

Las redes complejas de mutualismos no son colecciones casuales de especialistas y de generalistas. La evolución y la coevolución dan forma a estas interacciones multi-especie de manera predecible, sin importar la composición de especies exacta o el ecosistema, apuntando hacia una más manejable teoría coevolutiva dentro de las redes mutualistas complejas. Los estudios sobre redes complejas de mutualismos son un aspecto novel de la biología coevolutiva y de la ecología de comunidades y abarcan procesos que dan forma a la diversidad biológica a través de grandes escalas geográficas y temporales. También son una realización creciente de que mucha de la diversificación de la vida es so- 


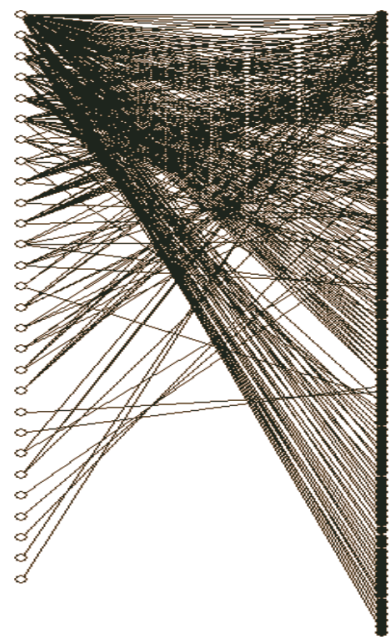

$\mathbf{L M}$
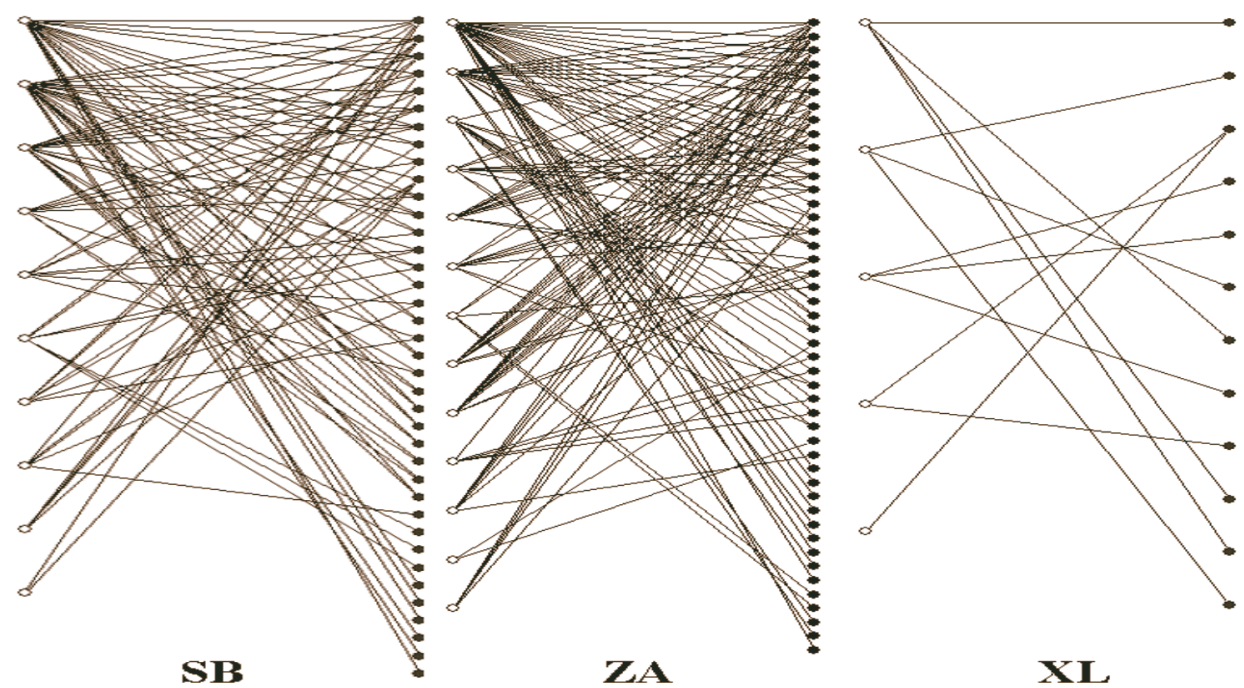

Figura 2. Redes hormiga (círculos)-planta con nectarios extraflorales (puntos negros). Las tres de la izquierda presentaron encajamiento significativo (ver GUIMARAEES et al., 2006).

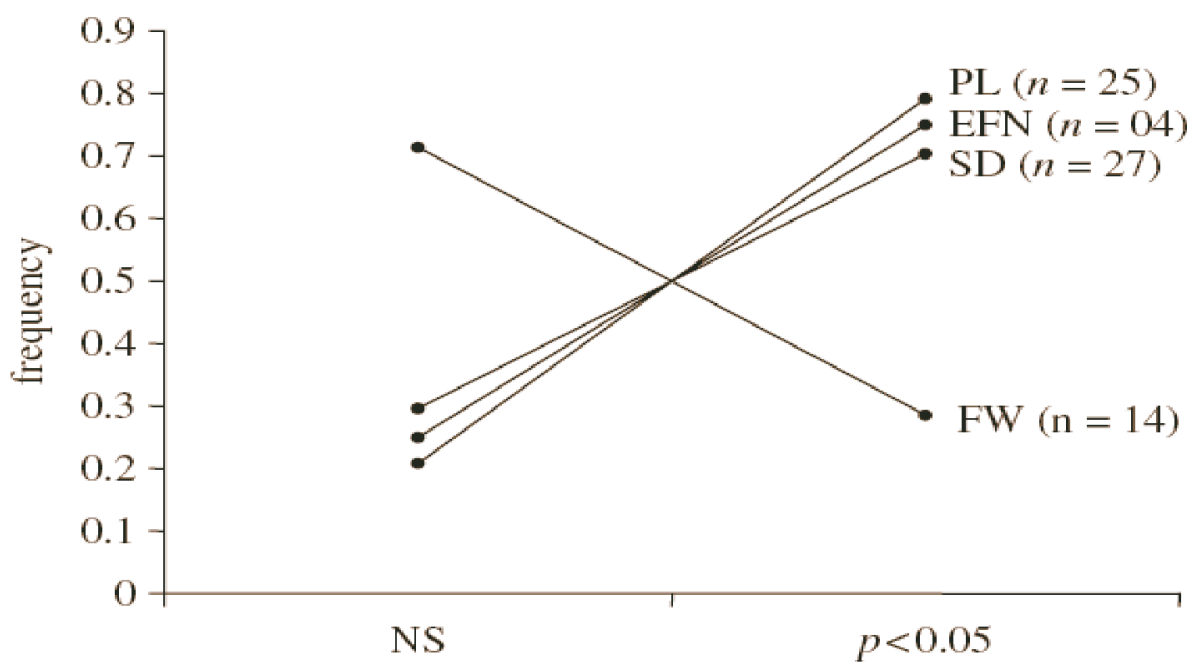

Figura 3. Proporción de encajamiento significativo encontrado en diferentes tipos de interacciones. PL, polinización; EFN, nectarios extraflorales; SD, dispersión de semillas y FW, red antagónica. NS, encajamiento no significativo y $p>0.05$ = encajamiento significativo. Entre paréntesis el número de redes analizadas (ver GUIMARÃES et al. 2006).

bre la diversificación de las interacciones a través de coevolución continua (BASCOMPTE et al., 2006; THOMPSON, 2005, 2006).

Estudios recientes van más allá de sólo considerar la presencia o ausencia de interacción entre las especies de una comunidad y se está encontrando que los análisis cuantitativos reflejan más adecuadamente propiedades de las redes de interacciones que los intentos cualitativos previos y son más robustos contra las variaciones en intensidad de muestreo, tamaño de la red y simetría (BLÜTHGEN et al., 2006). Estos índices servirán para ampliar nuestro conocimiento sobre los patrones de especialización dentro y entre redes desde un punto de vista amplio de las interacciones interespecíficas (BLÜTHGEN et al., 2006). 
Finalmente, las herramientas y las posibilidades que ofrece el análisis de redes para el estudio de la organización de las comunidades bióticas ha sido muy poco explorado (p.e., GUIMARÃES et al., 2005; VÁZQUEZ et al., 2005; BLÜTHGEN et al., 2006), lo que representa una avenida abierta a la investigación usando grandes conjuntos de interacciones entre especies (p.e., BASCOMPTE et al., 2003). Esto mejorará nuestro entendimiento acerca de la organización de las comunidades y, en particular, a explorar cómo diferentes tipos de interacciones dan forma a las comunidades (OLESEN \& JORDANO, 2002; BASCOMPTE \& JORDANO, 2006). El patrón anidado (encajado) que se obtiene de redes de mutualismos altamente estructuradas es importante para tener un mejor entendimiento de los patrones de coevolución en comunidades ricas en especies, las cuales no pueden ser reducidas a pares de especies coevolucionando ni a ensambles difusos interaccionando al azar (JORDANO et al., 2003; THOMPSON, 2005; BASCOMPTE \& JORDANO, 2006). Las implicaciones de esta aproximación a la estructura de las redes es importante para los estudios de coevolución, ecología de comunidades y ecología de la conservación (BASCOMPTE \& JORDANO, 2006). Se espera que un número mayor de estudios pronto utilicen esta visión de las comunidades con el fin de poder comparar diferentes tipos de comunidades, incluyendo ambos su organización interna y su organización geográfica, y poder aplicar los resultados con fines de conservación (RICO-GRAY \& OLIVEIRA, en prensa).

\section{REFERENCIAS}

ANDERSEN, A.N. \& J.D. Majer. 2004. Ants show the way down under: invertebrates as bioindicators in land management. Front. Ecol. Environ. 2:291-298.

BASCOMPTE, J., P. Jordano, C.J. Melián \& J.M. Olesen. 2003. The nested assembly of plant-animal mutualistic networks. Proc. Natl. Acad. Sci., USA 100:9383-9387.

BASCOMPTE, J. \& P. Jordano. 2006. The structure of plant animal mutualistic networks. En: M. Pascual \& J.A. Dunne (eds.), Food Webs as Complex Adaptative Networks: liking structure to dyamics. Oxford University Press, Oxford. Pp. 143-159.
BASCOMPTE, J., P. Jordano, \& J.M. Olesen. 2006. Asymmetric coevolutionary networks facilitate biodiversity maintenance. Science 312:431-433.

BLÜTHGEN, N., F. Menzel \& N. Blüthgen. 2006. Measuring specialization in species interaction networks. BMC Ecology 6:9 (doi:10.1186/14716785-6-9).

GUIMARÃES Jr., P.R., M.A.M. de Aguiar, J. Bascompte, P. Jordano \& S.F. dos Reis. 2005. Random initial condition in small Barabasi-Albert networks and deviations from the scale-free behavior. Physical Rev. E 71, art. no. 037101.

GUIMARÃES Jr., P.R., V. Rico-Gray, S.F. dos Reis \& J.N. Thompson. 2006. Asymmetries in specialization in ant-plant mutualistic networks. Proc. R. Soc. B 273:2041-2047.

HERRERA, C.M. 2005. Plant generalization on pollinators: species property or local phenomenon? Am. J. Bot. 92:13-20.

JORDANO, P. 1987. Patterns of mutualistic interactiones in pollination and seed dispersal: connectance, dependence asymmetries, and coevolution. Am. Nat. 129:657-677.

JORDANO, P., J. Bascompte y J.M. Olesen. 2003. Invariant properties in coevolutionary networks of plant-animal interactions. Ecol. Lett. 6:69-81.

OLESEN, J.M. y P. Jordano. 2002. Geographic patterns in plant-pollinator mutualistic networks. Ecology 83:2416-2424.

RICO-GRAY, V. \& P.S. Oliveira. 2007. The Ecology and Evolution of Ant-Plant Interactions. University of Chicago Press, Chicago (en prensa).

RUDGERS, J.A. \& S.Y. Strauss. 2004. A selection mosaic in the facultative mutualism between ants and wild cotton. Proc. R. Soc. B 271:2481-2488.

THOMPSON, J.N. 1994. The Coevolutionary Process. University of Chicago Press, Chicago.

THOMPSON, J.N. 2005. The Geographic Mosaic of Coevolution. University of Chicago Press, Chicago.

THOMPSON, J.N. 2006. Mutualistic webs of species. Science 312:372-373.

VÁZQUEZ, D.P. y M.A. Aizen. 2004. Asymmetric specialization: a pervasive feature of plant-pollinator interactions. Ecology 85:1251-1257.

VÁZQUEZ, D.P., W.F. Morris \& P. Jordano. 2005. Interaction frequency as a surrogate for the total effect of animal mutualists on plants. Ecol. Lett. 8:1088-1094. 